\title{
RentabiLean: Modelo de exploración del efecto de iniciativas Lean sobre la rentabilidad financiera
}

\section{RentabiLean: Exploration model of the effect of Lean initiatives on financial profitability}

Pérez-Rave Jorge

Universidad de Antioquia

Departamento de Ingeniería Industrial

Grupo de Investigación Gestión de la Calidad

Correo: jiprave@jiprave.com

Ocampo Fernando

Universidad de Antioquia

Departamento de Ingeniería Industrial

Grupo de Investigación Gestión de la Calidad

Correo: grupogestiondecalidad@udea.edu.co

\author{
Restrepo Guillermo \\ Universidad de Antioquia \\ Departamento de Ingeniería Industrial \\ Grupo de Investigación Gestión de la Calidad \\ Correo: grestreg@une.net.co
}

\section{Resumen}

Una de las dificultades en la adopción del Lean es conocer sus impactos financieros. El objetivo fue proponer un modelo cuantitativo que relacione tres indicadores operativos (impactados comúnmente con iniciativas Lean), con métricas financieras. El estudio se ejecutó en tres fases: la primera, permitió obtener un panorama general del tema, la segunda, se enfocó en la desagregación inicial de métricas financieras, a fin de facilitar la conexión posterior con variables operativas y la tercera, siguió la desagregación de variables hasta incorporar: tiempo de ciclo del proceso, proporción de calidad interna y horas para mantenimiento-reparaciones. Se aportan cinco elementos fundamentales: 1) un diagrama que retrata, en forma gráfica, las ecuaciones simultáneas, así como los efectos directos e indirectos, 2) expresiones que expresan la Utilidad Neta, los Activos y el ROA en términos de los tres indicadores de "piso", 3) expresiones de los efectos de dichos indicadores, 4) caso ilustrativo y resultados de aplicación que facilitan pruebas y comparaciones futuras, 5) una base para explorar los impactos de otros sistemas de mejora, con incidencia sobre los tres indicadores utilizados.

Descriptores: RentabiLean, impactos del Lean, costeo Lean, Lean en finanzas, modelo financiero del Lean.

\begin{abstract}
One of the difficulties in adopting the Lean is knowing its financial impacts. The objective was to propose a quantitative model that relates three operational indicators (commonly impacted with Lean initiatives), with financial metrics. The study was carried out in three phases: The first allowed an overview of the topic, the second one focused on the initial disaggregation of financial metrics, in order to facilitate the subsequent connection with operative variables, in the third, the disaggregation of variables followed until incorporating: process cycle time, internal quality ratio and hours for maintenance-repairs. It provides five fundamental elements: 1) A diagram that graphically portrays simultaneous equations as well as direct and indirect effects, 2) Expressions expressing Net Profit, Assets and ROA in terms of the three "floor" indicators, 3) Expressions of the effects of these indicators, 4) Illustrative case and application results that facilitate future tests and comparisons, 5) A basis for exploring the impacts of other improvement systems, with an impact on the three indicators used.
\end{abstract}

Keywords: RentabiLean, Lean impacts, Lean costing, Lean in finance, Lean financial model. 


\section{INTRODUCCIÓN}

La manufactura esbelta (Lean Manufacturing) posibilita generar cambios de mejora en la calidad, los tiempos de entrega, la reducción de costos, entre otros, (Elsukova, 2015; En-Nhaili et al., 2015). Esto se logra, a partir del capital intelectual, sobre la base de empoderamiento, poca inversión y pequeños cambios sistemáticos para reducir el desperdicio. Hoy en día, son cada vez más las empresas que buscan operar bajo iniciativas Lean, pues son reconocidas como el camino más cercano para obtener desempeños de clase mundial (Mamat et al. 2015). A pesar de ello, el avance de esta corriente no ha ido a la par con los sistemas contables de soporte, declarándose aún como un gap de estudio (Fullerton et al., 2014; Chiarini 2012; De Arbulo y De Basurto, 2007). Por ejemplo, los efectos del Lean son simples y tradicionales de expresar, bajo la comparación de "antes" vs "después" en los indicadores de "piso" (Ej: tasas de calidad, tiempos de producción, etcétera), pero llevarlos a impactos financieros, prioritarios para la alta gerencia, es complejo y es menos frecuente (Büyüközkan et al., 2015; Timm, 2015; Arbulo y Basurto, 2007). Más aún, desde un punto de vista práctico, la escases de un puente entre dichos efectos y los resultados financieros, pueden verse como un antecedente del bajo compromiso gerencial con el tema.

Por lo expuesto, el objetivo de este estudio fue explorar posibles respuestas a la siguiente pregunta: ¿Cuál es la naturaleza cuantitativa de las relaciones entre indicadores de nivel operativo, susceptibles de impactar mediante iniciativas Lean (tiempos de ciclo, calidad interna, tiempos de mantenimiento-reparaciones), y la rentabilidad financiera (ROA)?

El alcance del estudio, como se nota en la pregunta anterior, está delimitado a tres de los indicadores operativos, susceptibles de ser impactados a través de prácticas Lean (evidencias de impactos sobre estos indicadores, pueden verse en: Zhang et al., 2016; Dandin y Mench, 2015; Dharun y Ganesh, 2015). En cuanto a la expresión de la rentabilidad, se ha delimitado al ROA (Rentabilidad del Activo); entendida como la relación entre las utilidades y los activos de la organización en el ejercicio de sus actividades (Githman, 1997). Igualmente, se interpreta como la capacidad de los activos para generar utilidades, y se expresa como el cociente entre la utilidad neta - también llamada beneficio neto después de impuestos (Lesakova, 2007), o resultados de operación (Gheorhe, 2013) —, y los activos de la empresa.

Adicionalmente, acorde con la pregunta en estudio, el alcance del producto no es un sistema de costeo, sino un modelo cuantitativo que posibilita relacionar los in- dicadores operativos de interés con la rentabilidad financiera, a fin de explorar, en niveles superiores, los impactos de las acciones de "piso". Esto le apunta también a la necesidad de vincular al estudio del tema otras perspectivas, que posibiliten una mejor comprensión de las complejidades subyacentes (Elsukova, 2015; Monden y Wang, 2015; Chiarini, 2012).

El resto del artículo está organizado así: procedimiento, desarrollo del modelo (ecuaciones y representación gráfica), caso de ilustración (simulado) con discusión de resultados. Luego están las conclusiones y los trabajos futuros.

\section{Estudios Relacionados}

De Arbulo y Fortuy (2010) revisan técnicas de costeo aplicadas al Lean. Una vez más, se advierte sobre la necesidad de nuevos paradigmas de costeo. También, exponen paso a paso la aplicación de un método de costeo basado en actividades para estimar costos del producto y del desperdicio, así como los ahorros operativos. Además, concluyen que no hay consenso sobre cómo las prácticas contables deberían adoptarse y desarrollarse para soportar el Lean.

Chiarini (2012), argumenta que hay incompatibilidad entre el Lean y la contabilidad tradicional. Además, discute si el costeo ABC y el Value Stream Costing (VSC) son útiles para escenarios Lean. Encuentra varias limitaciones, entre ellas, que el VSC no necesariamente resulta adecuado para Pymes.

Khataie y Bulgak (2013) proponen un modelo de costeo de la calidad, basado en actividades y en simulación dinámica de sistemas, que puede ayudar a la gerencia a monitorear los efectos de los cambios de mejora en el tema. Vale anotar que este se orienta fundamentalmente a la componente de costos.

Fullerton et al. (2014), recalcan la necesidad de alinear la gestión de contabilidad y el Lean, señalando que este no debe limitarse a mejoramientos locales, sino que debe tomarse de forma holística como estrategia de negocio y retribuir en la mejora global de la empresa. Usando datos de encuestas y modelos de ecuaciones estructurales, concluyen que las prácticas de gestión contable sistemáticas y alineadas con el Lean, se relacionan de forma positiva con el desempeño del sistema.

Timm (2015), señala que los sistemas de gestión contable no han logrado adaptarse a los cambios vertiginosos de los sistemas de producción moderna, concretamente, bajo principios de Lean Manufacturing. Adicionalmente, concluye que hay poca investigación cuantitativa previa sobre la contabilidad del Lean, y aporta nueva información sobre antecedentes de la im- 
plementación de prácticas como el Value Stream Costing, usando datos de encuestas.

Elsukova (2015) también llama la atención sobre la necesidad de nuevos enfoques contables que soporte el Lean. Expone dos métodos de gestión contable (Lean Accounting y Throughput Accounting) y propone un enfoque integrador. Concluyen que esta propuesta puede aportar información más efectiva sobre costos y resultados en la empresa moderna, que cuando dichos métodos se utilizan por separado.

Büyüközkan et al. (2015) evalúan la incidencia de varias combinaciones de técnicas Lean sobre el desempeño financiero y no financiero, usando Redes Bayesianas. Advierte que pocos estudios han validado la incidencia del Lean sobre el desempeño empresarial. Bajo casos de simulación, concluye que es evidente el impacto significativo del Lean sobre métricas de desempeño no financiero (calidad, fiabilidad,...), pero también, que es muy reducido el efecto sobre las variables financieras. No obstante, reconoce las limitaciones del caso simulado y, por tanto, no pueden generalizarse los hallazgos, por lo que sugiere la replicación del estudio.

Monden y Wang (2015) examinan varias medidas de desempeño del Lean, que pueda tener relación con métricas financieras, y proponen otras nuevas. Diferencian entre tres niveles organizacionales: operacional, táctico y estratégico. Para este último, recalcan la necesidad de métricas que se relacionen con la rentabilidad, entre otros. Plantean que el ROA, como medida tradicional, puede utilizarse para medir los efectos del Lean, debido a que este, al llevar a reducir el Lead Time, merma los inventarios $\mathrm{y}$, por consiguiente, se afectan los activos. Básicamente, los aportes de este trabajo se sustentan en impactos sobre el Lead Time y los inventarios.

En general, la comunidad académica sigue recalcando que el Lean y las prácticas contables continúan desarticulados, $y$, por lo mismo, contribuye desde diversas perspectivas. No obstante, por lo emergente de este campo, ninguno de los métodos se asume como el más adecuado; cada estudio realiza valiosas contribuciones y abre paso a versiones mejoradas. Además, sus alcances, tradicionalmente se han enfocado en la componente de costos, más asociados con niveles tácticos que con intereses de la alta gerencia. A su vez, los pocos estudios que han abordado concretamente métricas como la rentabilidad, sugieren nuevas métricas o delimitan el alcance a impactos producidos por la reducción del Lead Time o inventarios. Sin embargo, no se encontraron antecedentes de desagregación de estas métricas, concretamente del ROA, los Activos y las
Utilidades, para expresarlos en términos de indicadores de piso, propios de calidad interna, tiempos de ciclo y horas de mantenimiento-reparaciones.

\section{Procedimiento}

El estudio se llevó a cabo en tres fases. La primera, tuvo como objetivo obtener un panorama general del costeo del Lean y de métricas financieras de la alta gerencia. La segunda fase se enfocó en una primera desagregación de métricas financieras, a fin de facilitar la conexión posterior con variables cercanas a niveles operativos. La tercera fase partió de los resultados de la fase anterior, para continuar desagregando hasta incorporar indicadores de "piso", que comúnmente se impacten mediante iniciativas Lean. Dado el alcance, dichos indicadores, fueron: tiempo de ciclo de la línea de producción (mayor tiempo de ciclo entre los centros de trabajo, es decir, la restricción), proporción de calidad interna (sin asumir reprocesos; los productos defectuosos se desechan), y horas dedicadas a mantenimiento y reparaciones (sin asumir acciones preventivas, solo correctivas).

Para llevar a cabo la fase 1, se recurrió a la lectura y análisis de documentos reportados por Google Scholar y Scopus, así como a la consulta del Plan Único de Cuentas (en Colombia, PUC, 2016). La fase 2 partió del ROA y sus componentes (Utilidad neta, numerador; Activo, denominador). Luego, similar a una estructura jerárquica, cada componente se desagregó en otras expresiones, dando lugar a nuevas variables, hasta comenzar a encontrar métricas cercanas a las operaciones, por ejemplo, inventario, costos unitarios, mantenimiento. Con base en la fase 1 y apoyados en el PUC, así como en la experiencia y en procesos de deducción, en la Tabla 1 se presenta la desagregación inicial del ROA, arrojada por la fase 2.

En la Tabla 1 se incluyó el término $a_{j}$ (por ejemplo: $a_{3}=$ gastos operacionales de ventas), que simboliza uno o más componentes financieros asumidos en este estudio con baja relación con indicadores en la rutina operativa, que generalmente se impactan de forma directa por iniciativas Lean (en procesos manufactureros). La fase 3 tuvo como herramienta de apoyo un esquema inspirado en los diagramas Path (usado en Modelos de Ecuaciones Estructurales), estos son útiles para representar las diferentes relaciones simultáneas entre las variables. Todo ello facilita la comprensión del fenómeno y la identificación de efectos directos e indirectos hasta llegar a los indicadores operativos objeto de estudio. Desde luego, al ser un elemento guía, tuvo sus adaptaciones, que se explican en el apartado de resultados. 
Tabla 1. Desagregación inicial de variables financieras, fase 2

\begin{tabular}{|c|c|c|c|}
\hline Notación & Fórmulas & Observaciones & Desagrega \\
\hline $\mathrm{UN}$ & $\begin{array}{l}\text { (1) UAIR - (IR + RL) } \\
\text { (2) UAIR - a1 }\end{array}$ & $\begin{array}{l}\text { UN (Utilidad neta); UAIR (Utilidad antes } \\
\text { de IR y RL); IR (Impuesto de renta); RL } \\
\text { (Reserva legal); a1: (IR + RL) }\end{array}$ & UAIR \\
\hline UAIR & $\begin{array}{l}\text { (3) } \mathrm{UO}+\text { INO - GNO } \\
\text { (4) } \mathrm{UO}+\mathrm{a} 2\end{array}$ & $\begin{array}{l}\text { UO (Utilidad operacional); INO (Ingresos } \\
\text { no operacionales); GNO (Gastos no } \\
\text { operacionales); a2: (INO - GNO) }\end{array}$ & $\mathrm{UO}$ \\
\hline $\mathrm{UO}$ & (5) $\mathrm{VN}-\mathrm{CMV}-\mathrm{GO}$ & $\begin{array}{l}\text { VN (Ventas netas); CMV (Costo de } \\
\text { la mercancía vendida); GO (Gastos } \\
\text { operacionales) }\end{array}$ & $\mathrm{VN}, \mathrm{CMV}, \mathrm{GO}$ \\
\hline $\mathrm{VN}$ & (6) $\mathrm{V}-\mathrm{DEV}$ & V (Ventas); DEV (Devoluciones en ventas) & V, DEV \\
\hline $\mathrm{V}$ & (7) preu $\times$ UV & $\begin{array}{l}\text { preu (Precio de unidad vendida); UV } \\
\text { (Unidades vendidas) }\end{array}$ & En fase 3 \\
\hline DEV & (8) CUDEV x UDEV & $\begin{array}{l}\text { CUDEV (Costo de unidad devuelta); } \\
\text { UDEV (Unidades devueltas) }\end{array}$ & En fase 3 \\
\hline CMV & (9) CUMV x UV & $\begin{array}{l}\text { CUMV (Costo unitario de mercancía } \\
\text { vendida) }\end{array}$ & En fase 3 \\
\hline GO & (10) GOA + a3 & $\begin{array}{l}\text { GOA (Gastos operacionales de } \\
\text { administración); a3: (Gastos operacionales } \\
\text { de ventas: GOV) }\end{array}$ & GOA \\
\hline GOA & (11) MYR + a4 & $\begin{array}{l}\text { MYR (Mantenimiento y reparaciones: } \\
\text { terrenos, equipo de oficina, maquinaria } \\
\text { y equipo, etcétera); a4: (Arrendamientos, } \\
\text { seguros, adecuación e instalación, } \\
\text { impuestos, depreciación, honorarios, } \\
\text { servicios, gastos legales, diversos, } \\
\text { provisiones, etcétera Y sueldos, jornales, } \\
\text { horas extras y recargos, comisiones, } \\
\text { viáticos, incapacidades, auxilios, } \\
\text { prestaciones sociales, bonificaciones, } \\
\text { dotación y suministros, seguros, etcétera, } \\
\text { de personal de administración) }\end{array}$ & En fase 3 \\
\hline \multirow[t]{2}{*}{ A } & $\begin{array}{l}(12) \mathrm{I}+\mathrm{OAC}+\mathrm{AF} \\
+\mathrm{AD}+\mathrm{OA}\end{array}$ & $\begin{array}{l}\text { A (Activos); I (Inventario); OAC (Otros } \\
\text { activos circulantes); AF (Activos fijos); } \\
\text { AD (Activos diferidos); OA (Otros } \\
\text { activos) }\end{array}$ & \multirow[t]{2}{*}{ En fase 3} \\
\hline & (13) $\mathrm{I}+\mathrm{a} 5$ & $\mathrm{a} 5:(\mathrm{OAC}+\mathrm{AF}+\mathrm{AD}+\mathrm{OA})$ & \\
\hline
\end{tabular}

\section{Desarrollo del modelo}

En la Tabla 2 se expone la desagregación final de las variables que arrojó la fase 2 (ver Tabla 1). Nótese que se llegó hasta variables directamente observables en los procesos operativos, comúnmente afectadas con iniciativas Lean, como Tiempos de Ciclo del Proceso (TCL), Proporción de Calidad Interna (PCALI) y Número de Horas de
Mantenimiento y Reparaciones (NHMYR). Cabe anotar que las variables con asterisco $\left(^{*}\right)$ en la Tabla 2, tienen, en este caso, dos formas de representarse.

Tomando en consideración la Tabla 2, en la Figura 1 se presenta el diagrama de relaciones empleado para obtener un panorama global de las ecuaciones que interactúan entre sí, así como de los efectos directos e indirectos resultantes. 
Tabla 2. Desagregación final hasta indicadores operativos comúnmente impactados por iniciativas Lean, fase 3

\begin{tabular}{|c|c|c|}
\hline Notación & Fórmulas & Observaciones \\
\hline CUMV & $\begin{array}{l}\text { (14) CUMDUC } \\
+ \text { CUMODUC + a6 }\end{array}$ & $\begin{array}{l}\text { CUMDUC (Costo unitario de material directo necesario para } \\
\text { obtener unidades conformes); CUMODUC (Costo unitario } \\
\text { de mano de obra directa para obtener unidades conformes); } \\
\text { a6 (Costos unitarios indirectos de fabricación) }\end{array}$ \\
\hline CUMDUC & $\begin{array}{l}\text { (15) a7 - CUMD } \\
\text { x PCALI }\end{array}$ & $\begin{array}{l}\text { CUMD (Costo unitario de material directo); PCALI } \\
\text { (Proporción de calidad interna) }\end{array}$ \\
\hline a7 & (16) $2 \times$ CUMD & Factor constante 7 \\
\hline CUMODUC* & $\begin{array}{l}\text { (16a) TCL } \\
x \text { fc.modlyncali }\end{array}$ & $\begin{array}{l}\text { TCL (tiempo de ciclo de la línea); fc.modlyncali (factor de } \\
\text { costo de mano de obra directa de la línea y no calidad) }\end{array}$ \\
\hline fc.modlyncali & $\begin{array}{l}(17) \text { npmodl } \\
x \text { chmod } \\
x(2-\text { PCALI })\end{array}$ & $\begin{array}{l}\text { npmodl (número de personas de mano de obra directa } \\
\text { en la línea); chmod (Costo de una hora de una persona } \\
\text { perteneciente a la mano de obra directa) }\end{array}$ \\
\hline CUMODUC $^{*}$ & $\begin{array}{l}(16 b) \text { a } 8 \\
- \text { fc.tcymodl x PCALI }\end{array}$ & \\
\hline fc.tcymodl & $\begin{array}{l}\text { (18) TCL } x \text { chmod } \\
x \text { npmodl }\end{array}$ & de obra directa de la línea) \\
\hline a8 & (19) 2 x fc.tcymodl & Factor constante 8 \\
\hline UV & (20) UTER x fuv & $\begin{array}{l}\text { UTER (Unidades terminadas); fuv (factor de unidades } \\
\text { vendidas en relación a las terminadas) }\end{array}$ \\
\hline UTER $^{* *}$ & $\begin{array}{l}\text { (21a) tdis } x \text { ftccali } \\
\text { x TCL }\end{array}$ & $\begin{array}{l}\text { tdis (Tiempo disponible de producción); ftccali (factor de } \\
\text { tiempo de ciclo y calidad interna) }\end{array}$ \\
\hline ftccali & (22) PCALI / TCL ${ }^{2}$ & \\
\hline $\mathrm{UTER}^{* *}$ & $\begin{array}{l}(21 \mathrm{~b}) \text { tdis } \mathrm{x} \mathrm{ftc} \\
\mathrm{x} \text { PCALI }\end{array}$ & ftc (Factor de tiempo de ciclo) \\
\hline $\mathrm{ftc}$ & (23) 1 / TCL & \\
\hline$I(j)$ & (24) $\mathrm{CI}(\mathrm{j}) \times \mathrm{UI}(\mathrm{j})$ & $\begin{array}{l}\mathrm{CI}(\mathrm{j}) \text { (Costo de una unidad de inventario j); UI(j) (Unidades } \\
\text { de inventario j). Con j: PT (Producto terminado), PP } \\
\text { (Producto en proceso), MP (Materia prima) }\end{array}$ \\
\hline UIMP & (25) fmp x UTER & $\begin{array}{l}\text { fmp (factor de cantidad de materia prima en relación a las } \\
\text { unidades terminadas) }\end{array}$ \\
\hline UIPP & (26) fpp x UTER & $\begin{array}{l}\text { fpp (factor de cantidad de producto en proceso en relación a } \\
\text { las unidades terminadas) }\end{array}$ \\
\hline UIPT & (27) $(1$ - fuv) x UTER & Definidos en (20). \\
\hline MYR & (28) chmyr x NHMYR & $\begin{array}{l}\text { chmyr (Costo de una hora de mantenimiento y } \\
\text { reparaciones); NHMYR (Número de horas de } \\
\text { mantenimiento y reparaciones) }\end{array}$ \\
\hline
\end{tabular}


Para leer tal diagrama, basta con enfocar alguno de los recuadros. Por ejemplo, el que tiene las siglas UN representa la Utilidad Neta. Luego, se deben considerar las flechas que llegan al recuadro, que en este caso son dos, una que parte de UAIR (Utilidad Antes de Impuestos y Reservas) y otra que parte de $\mathrm{a}_{1}$. Así, la forma de deducir la expresión funcional, es la misma ecuación 2 (véase Tabla 1), de naturaleza lineal. La expresión ubicada sobre cada flecha en el diagrama (Figura 1), corresponde al factor multiplicativo que acompaña a la variable de interés, en este caso puntual: +1 y -1 ; pero, por ejemplo, para la variable " $\mathrm{V}$ ", a la que solo llega una flecha que tiene su origen en UV, el factor multiplicativo es "preu".

Nótese que la Figura 1 ilustra una estructura jerárquica que se va desagregando de derecha (ROA) a izquierda (indicadores operativos de interés, expresados como $\mathrm{w}_{\mathrm{i}}$ ). Sin embargo, este diagrama tiene una particularidad, y es que, tanto la variable UTER como CUMODUC, pueden plantearse de dos formas diferentes. Una es en términos del Tiempo de Ciclo de la Línea (TCL) y la otra en función de la Proporción de Calidad Interna (PCALI). Así, dependiendo del interés que se tenga, puede tomarse una ruta u otra para llegar al mismo resultado. Estos caminos alternos están representados en el diagrama de relaciones (Figura 1) por medio de la letra "ó" (con tilde).

Para comenzar a explorar los efectos de una determinada variable sobre métricas financieras, basta con seguir los caminos posibles desde ella hasta la variable financiera de interés y considerar las expresiones ubicadas sobre las fechas. Por ejemplo, el efecto del GOA sobre la Utilidad Neta (UN), se obtiene así

$\Delta U N(G O A)=1 \times(-1) \times 1 \times 1=-1$

Esto significa que, por cada unidad que aumente GOA, la utilidad neta cambia en -1 unidades monetarias. Véa- se lo simple que resultó deducir este efecto, a partir del diagrama (Figura 1), sin necesidad de despejar y reemplazar bajo las ecuaciones 1-5 y 10 de la Tabla 1. En general, esto resulta útil, sobre todo cuando se toma en consideración que, a medida que se desagregan nuevas variables (moverse hacia la izquierda en el diagrama), aumenta la cantidad de términos y relaciones $\mathrm{y}$, por tanto, incrementa la complejidad.

A través de este diagrama (figura 1), es posible observar de manera más completa las relaciones entre las tres variables de interés $\left(\mathrm{w}_{\mathrm{i}}\right)$ y el ROA. Cabe señalar que cuando se llega a la expresión del ROA, se está frente al cociente de dos funciones, para lo cual, el efecto de $\mathrm{w}_{\mathrm{i}}$ sobre él puede hallarse mediante la derivada de un cociente. En la Tabla 3 se exponen las expresiones para la Utilidad Neta, el Activo y el ROA en función de los indicadores operativos de estudio $\left(\mathrm{w}_{\mathrm{i}}\right)$. También, en la columna de especificaciones pueden verse, entre otros, los efectos deducidos; estos se interpretan como el cambio en la variable financiera ante un cambio unitario en $\mathrm{w}_{\mathrm{i}}$.

A partir de la Tabla 3, vale señalar que el tiempo de ciclo de la línea $\left(\mathrm{w}_{1}\right)$ tiene un efecto no lineal sobre la Utilidad Neta (UN), contrario a lo que se observa para la proporción de calidad interna $\left(\mathrm{w}_{2}\right)$ y el número de horas de mantenimiento y reparaciones $\left(w_{3}\right)$.

Como ejercicio de validación, se estudió la coherencia de la escala de valores que toman las variables resultantes, al considerar las expresiones que las definen. A modo de ejemplo, considérese las unidades de medida resultantes, al aplicar la ecuación (30) (Tabla 3)

\section{CASO DE ILUSTRACIÓN Y DISCUSIÓN DE RESULTADOS}

Supóngase una organización con una única línea de producción, compuesta por tres centros de trabajo en serie, caracterizada por los parámetros especificados en la Tabla 4.

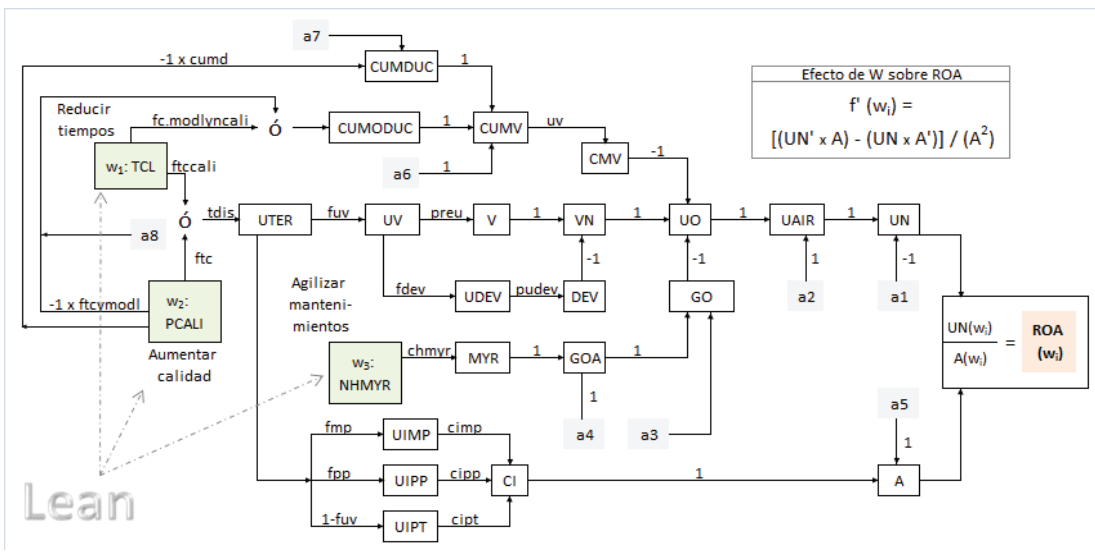

Figura 1. Path de RentabiLean (Modelo de relaciones entre medidas de operacionales y métricas financieras) 
Tabla 3. Modelo cuantitativo de variables financieras según $\mathrm{w}_{\mathrm{i}}$

\begin{tabular}{|c|c|c|}
\hline Medidas & Expresiones & Especificaciones \\
\hline$U N\left(W_{1}\right)$ & (30) $\left(b_{1} / w_{1}\right)+b_{2}$ & $\begin{array}{l}\text { (31) } b_{1}=P C A L I \times t \text { dis } \times \text { fuv } \times\left(\text { preu }-f d e v \times c u d e v a_{6}-\text { CUMDUC }\right) \\
\text { (32) } b_{2}=a_{2}-a_{1}-f u v \times t d i s \times P C A L I \times f c . m o d l y n c a l i-G O\end{array}$ \\
\hline$U N^{\prime}\left(W_{1}\right)$ & (33) $\left(-b_{1} / w_{1}^{2}\right)$ & Efecto de $w_{1}$ sobre la UN \\
\hline$U N\left(W_{2}\right)$ & (34) $b_{3} \times w_{2}^{2}+b_{4} \times w_{2}+b_{5}$ & $\begin{array}{l}\text { (35) } b_{3}=f t c \times t d i s \times f u v \times(f c . t c y m o d l+c u m d) \\
\text { (36) } b_{4}=f t c \times t d i s \times f u v \times\left(\text { preu }- \text { fdev } \times \text { cudev }-a_{8}-a_{6}-a_{7}\right) \\
\text { (37) } b_{5}=a_{2}-a_{1}-G O\end{array}$ \\
\hline$U N^{\prime}\left(W_{2}\right)$ & (38) $2 \times b_{3} \times w_{2}+b_{4}$ & Efecto de $w_{2}$ sobre la UN \\
\hline$U N\left(W_{3}\right)$ & (39) $b_{7}-b_{6} \times w_{3}$ & $\begin{array}{l}\text { (40) } b_{6}=\text { chmyr } \\
\text { (41) } b_{7}=V N-C M V-a_{1}+a_{2}-a_{3}-a_{4}\end{array}$ \\
\hline$U N^{\prime}\left(W_{3}\right)$ & $(42)-b_{6}$ & Efecto de $w_{3}$ sobre la UN \\
\hline$A\left(W_{1}\right)$ & $(43) a_{5}+\left(b_{8} \times P C A L I\right) / w_{1}$ & \\
\hline$A\left(W_{2}\right)$ & (44) $b_{8} \times f t c \times w_{2}+a_{5}$ & $(46) b_{8}=t d i s \times[f m p \times \operatorname{cimp}+f p p \times \operatorname{cipp}+\operatorname{cipt} \times(1-f u v)]$ \\
\hline$A\left(W_{3}\right)$ & (45) $a 5+b 8 \times U T E R / t d i s$ & \\
\hline$R O A\left(W_{\mathrm{i}}\right)$ & (47) UN $\left(w_{i}\right) / A\left(w_{i}\right)$ & Cociente de dos funciones \\
\hline$R O A^{\prime}\left(W_{\mathrm{i}}\right)$ & $\begin{array}{l}(48)\left[U N^{\prime}\left(w_{i}\right) \times A\left(w_{i}\right)-U N\left(w_{i}\right) \times\right. \\
\left.A^{\prime}\left(w_{i}\right)\right] / A\left(w_{i}\right)^{2}\end{array}$ & Efecto de w sobre el ROA \\
\hline
\end{tabular}

Tabla 4. Parámetros supuestos para el caso de ilustración

\begin{tabular}{|c|c|c|c|}
\hline TCL (h/u) & 7 & chmyr (\$/h) & 200 \\
\hline npmodl (cant) & 3 & $\operatorname{cimp}(\$ / \mathrm{u})$ & 1200 \\
\hline cumd $(\$ / \mathrm{u})$ & 5 & $\operatorname{cipt}(\$ / \mathrm{u})$ & 8000 \\
\hline chmod (\$/ h_per) & 30 & a1 (\$/mes) & 80000 \\
\hline tdis (h/mes) & 1980 & a2 (\$/mes) & 30000 \\
\hline $\operatorname{cipp}(\$ / \mathrm{u})$ & 5000 & a3 (\$/mes) & 400000 \\
\hline $\mathrm{fmp}$ & 1.5 & a4 (\$/mes) & 800000 \\
\hline fuv & 0.8 & a5 (\$/mes) & 250000 \\
\hline fpp & 0.4 & a6 (\$/u) & 15 \\
\hline fdev & 0.05 & preu $(\$ / \mathrm{u})$ & 12000 \\
\hline cudev $(\$ / \mathrm{u})$ & 13000 & $\begin{array}{l}\text { NHMYR (h/ } \\
\text { mes) }\end{array}$ & 192 \\
\hline PCALI & 0.9 & & \\
\hline
\end{tabular}

Vale resaltar que la línea presenta tres supuestos adicionales. El primero, es que las horas dedicadas al mes para mantenimiento-reparaciones se deben, únicamen- te, a lo correctivo (no preventivo). Esto, ya que el modelo no está considerando posibles impactos a largo plazo, debidos a la prevención de fallos en máquinas o equipos. El segundo, es que independiente de cuánto cambien los TCL, PCALI y NHMYR, los factores multiplicativos permanecen constantes. Por ejemplo, el factor de unidades vendidas (fuv) sigue de $80 \%$ por las unidades terminadas, independiente del escenario. Esto asume, por ejemplo, que mientras más capacidad (y producción), la participación en el mercado es la misma: 0.80 de los productos terminados, manejando un inventario de producto final de $20 \%$. El tercero, se refiere a que los productos defectuosos, detectados en el proceso, se desechan (no hay reprocesos).

Con los datos del caso de ilustración $\mathrm{y}$, haciendo uso de las Tablas 1-3, con apoyo de la figura 1 y Excel, es posible reproducir los resultados expuestos en la Tabla 5. Esta condensa, entre otros, los valores para ROA, Activos y Utilidad Neta ante diversos escenarios de prueba. En total, fueron 20 escenarios de cambio de los indicadores de "piso", pero por efectos de extensión del manuscrito, solo se presenta para 16 de ellos $\left(E\right.$. $\mathrm{w}_{1}$ (TCL), se muestra desde $3 \mathrm{~h} / \mathrm{u}$ hasta $18 \mathrm{~h} / \mathrm{u}$ ).

Otro ejercicio de consistencia de los resultados de las ecuaciones finales (Tabla 3 ), es verificar que estas expresiones, bajo las condiciones iniciales del caso de ilus- 
tración $\left(\mathrm{w}_{1}=7 \mathrm{~h} / \mathrm{u} ; \mathrm{w}_{2}=0.90 ; \mathrm{w}_{3}=192 \mathrm{~h} / \mathrm{mes}\right)$, lleven a los mismos resultados. Así, en la Tabla 5 puede verse, entre otras salidas, que en las condiciones iniciales (resultados en negrita) todos los valores coinciden (UN: \$ 877799; A: \$ 1624686; ROA: 0.54). De este modo, es viable asegurar las siguientes expresiones de balance

$$
\begin{aligned}
& \operatorname{UN}\left(w_{1}\right)=U N\left(w_{2}\right)=U N\left(w_{3}\right) \\
& A\left(w_{1}\right)=A\left(w_{2}\right)=A\left(w_{3}\right) \\
& \operatorname{ROA}\left(w_{1}\right)=\operatorname{ROA}\left(w_{2}\right)=\operatorname{ROA}\left(w_{3}\right)
\end{aligned}
$$

Otra situación útil como ejercicio de validación, es observar el comportamiento de las tres métricas financieras de interés ( $\mathrm{UN}, \mathrm{A}, \mathrm{ROA}$ ), ante cambios en los indicadores de "piso" $\left(\mathrm{w}_{\mathrm{i}}\right)$. $\mathrm{Al}$ respecto, sería de esperar un comportamiento consistente con lo que reporta la teoría y la experiencia. Para ello, en las Figuras 2 y 3 se presenta la evolución de estas métricas financieras, ante los 20 escenarios de variación de los indicadores de "piso" utilizados. En ese sentido, los tiempos de ciclo oscilaron entre $1 \mathrm{~h} / \mathrm{u}-20 \mathrm{~h} / \mathrm{u}$; la proporción de calidad interna (PCALI) desde 0.05 hasta 0.95; y las horas de mantenimiento y reparaciones (NHMYR), fueron desde $122 \mathrm{~h} /$ mes hasta $228 \mathrm{~h} / \mathrm{mes}$.

\begin{tabular}{|c|c|c|c|c|c|c|c|c|c|c|c|}
\hline & & & & & & & & & & & \\
\hline b2 & -1429534 & & & b4 & 2277566 & & & b7 & 916199 & & \\
\hline b1 & 16151335 & b8 & 10692000 & b3 & 143691 & & & b6 & 200 & & \\
\hline w1 & UN(w1) & $\mathrm{A}(\mathrm{w} 1)$ & $\mathrm{ROA}(\mathrm{w} 1)$ & w2 & $\mathrm{UN}(\mathrm{w} 2)$ & $\mathrm{A}(\mathrm{w} 2)$ & $\mathrm{ROA}(w 2)$ & w3 & UN(w3) & $\mathrm{A}(\mathrm{w} 3)$ & ROA(w3) \\
\hline 3 & 3954244 & 3457600 & 1.14 & 0.15 & -943532 & 479114 & -1.97 & 132 & 889799 & 1624686 & 0.5477 \\
\hline 4 & 2608299 & 2655700 & 0.98 & 0.2 & -827139 & 555486 & -1.49 & 138 & 888599 & 1624686 & 0.5469 \\
\hline 5 & 1800733 & 2174560 & 0.83 & 0.25 & -710028 & 631857 & -1.12 & 144 & 887399 & 1624686 & 0.5462 \\
\hline 6 & 1262355 & 1853800 & 0.68 & 0.3 & -592198 & 708229 & -0.84 & 150 & 886199 & 1624686 & 0.5455 \\
\hline 7 & 877799 & 1624686 & 0.54 & 0.35 & -473650 & 784600 & -0.60 & 156 & 884999 & 1624686 & 0.5447 \\
\hline 8 & 589382.5 & 1452850 & 0.41 & 0.4 & -354383 & 860971 & -0.41 & 162 & 883799 & 1624686 & 0.5440 \\
\hline 9 & 365058.4 & 1319200 & 0.28 & 0.45 & -234398 & 937343 & -0.25 & 168 & 882599 & 1624686 & 0.5432 \\
\hline 10 & 185599.1 & 1212280 & 0.15 & 0.5 & -113694 & 1013714 & -0.11 & 174 & 881399 & 1624686 & 0.5425 \\
\hline 11 & 38768.8 & 1124800 & 0.03 & 0.55 & 7728 & 1090086 & 0.01 & 180 & 880199 & 1624686 & 0.5418 \\
\hline 12 & -83589.8 & 1051900 & -0.08 & 0.6 & 129868 & 1166457 & 0.11 & 186 & 878999 & 1624686 & 0.5410 \\
\hline 13 & -187124 & 990215 & -0.19 & 0.65 & 252727 & 1242829 & 0.20 & 192 & 877799 & 1624686 & 0.5403 \\
\hline 14 & -275867.6 & 937343 & -0.29 & 0.7 & 376305 & 1319200 & 0.29 & 198 & 876599 & 1624686 & 0.5396 \\
\hline 15 & -352778.7 & 891520 & -0.40 & 0.75 & 500601 & 1395571 & 0.36 & 204 & 875399 & 1624686 & 0.5388 \\
\hline 16 & -420076 & 851425 & -0.49 & 0.8 & 625615 & 1471943 & 0.43 & 210 & 874199 & 1624686 & 0.5381 \\
\hline 17 & -479455.9 & 816047 & -0.59 & 0.85 & 751348 & 1548314 & 0.49 & 216 & 872999 & 1624686 & 0.5373 \\
\hline 18 & -532238 & 784600 & -0.68 & 0.9 & 877799 & 1624686 & 0.54 & 222 & 871799 & 1624686 & 0.5366 \\
\hline
\end{tabular}

Tabla 5. Resultados de aplicar el modelo al caso de ilustración

Tabla 5. Resultados de aplicar el modelo at caso de ilustración
De la figura 2a, se nota que las métricas financieras (UN y A) se perjudicaron de forma exponencial a medida que el tiempo de ciclo de la línea de producción $\left(\mathrm{w}_{1}\right)$ fue en aumento, es decir, mientras más se tardó la línea de producción en "gotear" sus productos (mayor tiempo de ciclo de los centros de trabajo: restricción). No obstante, vale recordar el supuesto de demanda de 0.8 de lo producido, independiente del escenario de simulación, lo cual representa que no hay agotamiento del mercado y que este se mantiene controlado en $20 \%$ de las unidades terminadas. De lo contrario, una reducción notable del tiempo de ciclo de la línea, podría generar una excesiva producción por encima de la demanda, conducir a altos costos de inventario y perjudicar la utilidad, entre otras variables. Otro aspecto por señalar, es que en alguno de los valores del tiempo de ciclo $\left(\mathrm{w}_{1}\right)$, los activos podrían ser equivalentes a la utilidad neta (en el caso de ilustración, cerca de TCL de 4 $\mathrm{h} / \mathrm{u}$ ) y, a partir de ahí los activos comienzan a superarla, lo cual conduciría a un menor ROA.

En relación con el comportamiento de la $\mathrm{UN}$ y el A, ante cambios en la proporción de calidad interna (Figura $2 b$ ), sobresalió un comportamiento lineal, a pesar de que las expresiones incorporaron términos cuadráticos (Tabla 3, ecuación 34). Este comportamiento, se debe a que los valores que toma $\mathrm{w}_{2}$ oscilan entre 0 y 1 , repre- 
sentando una proporción. Lo importante en este acercamiento con el tema, es que el comportamiento general fue consistente con lo que dicta la teoría: la calidad retribuye a un mejor rendimiento financiero. Es de anotar que las utilidades, en este caso puntual, crecieron a una tasa relativamente mayor que los activos a medida que aumentó la calidad interna. Asimismo, para esta situación de prueba, los activos siempre estuvieron por encima de la utilidad.

Respecto al número de horas de mantenimiento y reparaciones, el cambio en las utilidades no fue tan visible como ocurrió con los indicadores anteriores. De hecho, recuérdese que los activos se mostraron constantes ante cambios en $\mathrm{w}_{3}$, ya que no están incorporando tal indicador. Ahora bien, cuando se graficó de forma independiente las utilidades según $\mathrm{w}_{3}$ (Figura 2d), el comportamiento fue más visible (cambio de escala). Como era de esperar, a medida que se utilizaron más horas para mantenimiento y reparaciones (alcance: correctivo), los costos alusivos tendieron a perjudicar la utilidad, bajo un comportamiento lineal. Recuérdese también, que el caso de ilustración es una firma que solo realiza mantenimiento correctivo, por lo que no se están considerando beneficios, a largo plazo, de prevenir fallos en maquinaria y equipos, etcétera.

Pasando al ROA, en la Figura 3 se ilustra su comportamiento.

En la Figura 3a las variables están en la misma escala, en tanto, la Figura $3 b$ ha creado una escala secundaria para mejor visualización del ROA según $\mathrm{w}_{3}$ (mantenimiento y reparaciones). Véase, entonces, que los resultados, ante el caso de ilustración, fueron consistentes con lo que es de esperar:

1) La calidad lleva al mejoramiento de la rentabilidad.

2) A medida que aumenta el tiempo de ciclo de la línea, la rentabilidad se afecta negativamente (considerando el supuesto de inventario constante de $20 \%$ de las unidades terminadas).

3) Mientras más consumo de horas dedicadas a mantenimiento y reparaciones (bajo enfoque netamente correctivo) la rentabilidad se perjudica.
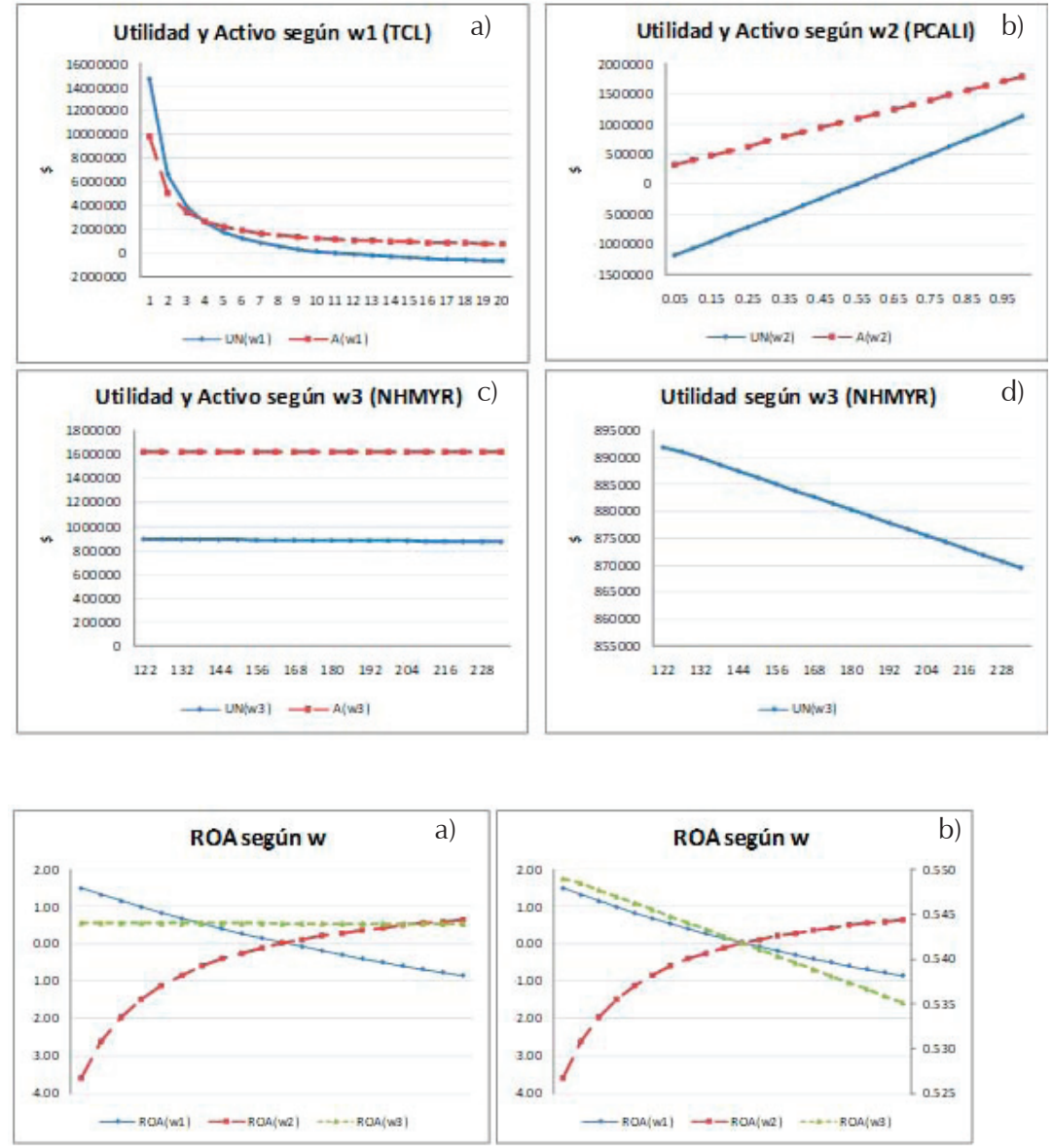

Figura 2. Utilidad Neta y Activos según $w_{1}$

Figura 3. a) ROA según wi, b) con escala secundaria 


\section{Conclusiones}

La principal contribución del estudio es proponer un nuevo modelo cuantitativo para explorar el efecto de iniciativas Lean sobre la rentabilidad financiera. Específicamente, de iniciativas que repercutan en al menos una de estas tres métricas de estudio: tiempo de ciclo de la línea de producción, proporción de calidad interna, número de horas de mantenimiento y reparaciones (con enfoque netamente correctivo). Este modelo aporta cinco elementos fundamentales. El primero, es el diagrama de relaciones, que de forma visual y simple ayuda a identificar el sistema de ecuaciones simultáneas implícito, así como efectos directos e indirectos. El segundo, son las ecuaciones como tal, explícitas, que retratan la Utilidad Neta (UN), los Activos (A) y el ROA en términos de los $\mathrm{w}_{\mathrm{i}}$. El tercero, corresponde a las ecuaciones de los efectos específicos de los $\mathrm{w}_{\mathrm{i}}$ sobre dichas métricas financieras (permaneciendo todo lo demás constante). El cuarto, es el caso de estudio y los resultados obtenidos, los cuales permiten realizar pruebas futuras y comparaciones de otros posibles modelos o agregaciones que vayan surgiendo. El quinto, se refiere a que este estudio constituye una base para explorar los impactos financieros de otros sistemas de mejora, que tengan incidencia sobre los indicadores de "piso" utilizados (tiempos de ciclo, calidad interna, y mantenimiento-reparaciones).

\section{LIMITACIONES Y TRABAJOS FUTUROS}

Este modelo, como primera aproximación, se limita a una serie de supuestos y a un solo caso de ilustración (simulado), los cuales, futuros estudios deberían superar. Por consiguiente, se requiere mayor evidencia para seguir probando su utilidad, usando la replicación del modelo en nuevos escenarios empresariales.

También, se sugiere nutrir el sistema de ecuaciones con nuevos indicadores operativos, susceptibles de impactar a través de Lean (Lead Time), así como de tipo financiero (rentabilidad del patrimonio). Adicionalmente, incrementar la complejidad, mediante la relajación de algunos de los supuestos utilizados con fines teóricos: participación constante en el mercado ( 0.80 de las unidades terminadas, lo que deriva en conservar $20 \%$ de inventario), productos defectuosos desechados (sin posibilidad de reprocesar), o mantenimiento/reparaciones netamente correctivo.

\section{RefERENCIAS}

Büyüközkan G., Kayakutlu G., Karakadılar İ. Assessment of lean manufacturing effect on business performance using Bayesian Belief Networks. Expert Systems with Applications, volumen 42 (número 19), 2015: 6539-6551.

Chiarini A. Lean production: Mistakes and limitations of accounting systems inside the SME sector. Journal of Manufacturing Technology Management, volumen 23 (número 5), 2012: 681700.

Dandin S. y Mench R. Development and implementation of Kaizen activities in an Automobile manufacturing firm. International Journal of Innovative Research in Advanced Engineering (IJIRAE), volumen 2 (número 6), 2015: 60-65.

De Arbulo P. y De Basurto P. Un modelo de gestión de costes para avanzar hacia la producción Lean. International Conference on Industrial Engineering \& Industrial Management-CIO 2007, pp. 1313-1322.

De Arbulo P. y Fortuny J. An accounting system to support process improvements: Transition to lean accounting. Journal of Industrial Engineering and Management, volumen 3 (número 3), 2010: 576-602.

Dharun K, Sakthi K. y Ganesh N. Cycle time reduction for T-shirt manufacturing in a Textile industry using lean tools, 2015 International Conference on Innovations in Information, Embedded and Communication Systems (ICIIECS), Coimbatore, 2015, pp. 1-6.

Elsukova T. Lean accounting and throughput accounting: An integrated approach. Mediterranean Journal of Social Sciences, volumen 6 (número 3), 2015: 83-87.

En-Nhaili A., Medaoui A., Bouami D. A new tool for maintenance performance measurement using value stream mapping and time-driven activity-based costing. International Journal of Process Management and Benchmarking, volumen 5 (número 2), 2015: 171-193.

Fullerton R., Kennedy F., Widener S. Lean manufacturing and firm performance: The incremental contribution of lean management accounting practices. Journal of Operations Management, volumen 32 (números 7-8), 2014: 414-428.

Gheorghe S. The Analysis of Profitability Indicators. Annals-Economy Series, (número 4), 2013: 132-138.

Gitman L. Fundamentos de administración financiera, México, Editorial OUP Harla, México, 1997, pp. 1077.

Khataie A.H. y Bulgak A. A cost of quality decision support model for lean manufacturing: activity-based costing application. International Journal of Quality \& Reliability Management, volumen 30 (número 7), 2013: 751-764.

Lesáková L. Uses and limitations of profitability ratio analysis in managerial practice, en: International Conference on Management, Enterprise and Benchmarking, June, 2007, pp. 1-2.

Mamat R., Md Deros B., Ab Rahman M., Omar M., Abdullah S. Soft lean practices for successful lean production system im- 
plementation in malaysia automotive smes: A proposed framework. Journal Teknologi, volumen 77 (número 27), 2015: 141-150.

Monden Y y Wang Z. Financial performance measures for the lean production system, en: Monden Y. y Minagawa Y. (Eds.). Lean Management of Global Supply Chain, 12, 2015, World Scientific, Singapore, pp. 147-158.

Plan Único de Cuentas, PUC, 2016, Colombia, http://puc.com.co

Timm P. Perceptions of value-stream costing and the effect on lean-accounting implementation, [dissertation submitted in partial fulfillment of the requirements for the degree of doctor of philosophy, management]. College of Management and Technology, Walden University, 2015, 184p.

Zhang A., Luo W., Shi Y., Chia S., Xavier Z. Lean and Six Sigma in logistics: a pilot survey study in Singapore. International Journal of Operations \& Production Management, volumen 36 (número 11), 2016: 1625-1643.

\section{Citación sugerida:}

\section{Citación estilo Chicago}

Pérez-Rave, Jorge, Fernando Ocampo, Guillermo Restrepo. RentabiLean: Modelo de exploración del efecto de iniciativas Lean sobre la rentabilidad financiera. Ingeniería Investigación y Tecnología, XIX, 01 (2018): 89-99.

\section{Citación estilo ISO 690}

Pérez-Rave J, Ocampo F, Restrepo G. RentabiLean: Modelo de exploración del efecto de iniciativas Lean sobre la rentabilidad financiera. Ingeniería Investigación y Tecnología, volumen XIX (número 1), eneromarzo 2018: 89-99.

\section{SemblanZas de lOS Autores}

Jorge Pérez-Rave. Ingeniero industrial por la Universidad de Antioquia (Colombia). Especialista en sistemas, especialista en estadística y magíster en ingeniería por la Universidad Nacional de Colombia. Máster en análiss y visualización de Big Data (UNIR). Investigador, consultor, emprendedor. Ha sido docente en el Departamento de Ingeniería Industrial de la Universidad de Antioquia y director del grupo de investigación gestión de la calidad.

Fernando Ocampo. Estudiante de ingeniería industrial por la Universidad de Antioquia. Emprendedor e integrante del Grupo Gestión de la Calidad.

Guillermo Restrepo. Ingeniero industrial por la Universidad de Antioquia, Economista por la misma universidad y especialista en finanzas de EAFIT. Es docente del Departamento de Ingeniería Industrial de la Universidad de Antioquia. 
DOI: http://dx.doi.org/10.22201/fi.25940732e.2018.19n1.008 